\title{
Bronquiolite obliterante com pneumonia em organização: relato de caso envolvendo um diagnóstico diferencial
}

\author{
Bronchiolitis obliterans organizing pneumonia: case report involving a differential diagnosis \\ Bronquiolitis obliterante con neumonía organizada: reporte de un caso con diagnóstico \\ diferencial
}

Recebido: 01/09/2021 | Revisado: 08/09/2021 | Aceito: 13/09/2021 | Publicado: 15/09/2021

\author{
Hiago Pereira Macedo \\ ORCID: https://orcid.org/0000-0002-6069-8784 \\ Universidade José do Rosário Vellano, Brasil \\ E-mail: macedo_hiago@yahoo.com.br \\ João Marcelo Lobo \\ ORCID: https://orcid.org/0000-0002-4053-0378 \\ Universidade José do Rosário Vellano, Brasil \\ E-mail: jmarcelo111@ hotmail.com \\ Matheus Henrique Defendi Barbosa \\ ORCID: https://orcid.org/0000-0003-2758-5902 \\ Universidade José do Rosário Vellano, Brasil \\ E-mail: matheushdbarboza@gmail.com \\ Mirian Nomura da Silva \\ ORCID: https://orcid.org/0000-0002-7672-5740 \\ Universidade José do Rosário Vellano, Brasil \\ E-mail: m.nomura93@ hotmail.com \\ Pollyana Rodrigues Reis \\ ORCID: https://orcid.org/0000-0001-6919-2483 \\ Universidade José do Rosário Vellano, Brasil \\ E-mail: pollyana_rodrigues_reis@ $@$ hotmail.com \\ Wandyk Allisson Bernardes Pereira \\ ORCID: https://orcid.org/0000-0001-9232-670X \\ Universidade José do Rosário Vellano, Brasil \\ E-mail: bwandyk@gmail.com \\ Cláudio Daniel Cerdeira \\ ORCID: https://orcid.org/0000-0002-7242-8028 \\ Universidade Federal de Alfenas, Brasil \\ E-mail: daniel.cerdeira.84@gmail.com \\ Fábio Antônio Gomes \\ ORCID: https://orcid.org/0000-0001-8610-0034 \\ Universidade José do Rosário Vellano, Brasil \\ E-mail: drfagomes@hotmail.com \\ Gérsika Bitencourt Santos Barros \\ ORCID: https://orcid.org/0000-0003-0849-2786 \\ Universidade José do Rosário Vellano, Brasil \\ E-mail: gersikasantos@unifenas.br
}

\begin{abstract}
Resumo
A bronquiolite obliterante com pneumonia em organização (BOPO) é uma entidade com sugestivo diagnóstico clínico e por imagem associado à evidência de tecido conjuntivo no lúmen dos espaços aéreos distais dos pulmões. Aqui abordamos um caso de BOPO e destacamos a importância do diagnóstico diferencial. Paciente do sexo masculino, 76 anos, ex-fumante (interrompeu o hábito há cerca de 30 anos), desenvolveu queixas de tosse copiosa e não produtiva, febre termometrada de $38{ }^{\circ} \mathrm{C}$ e emagrecimento sem alívio ao uso de fenoterol + brometo de ipatrópio. Paciente sem histórico recente de infecção, nega uso de drogas sistêmicas/inaladas, e não realizou viagens recentes. Relatou relevante histórico familiar para o câncer. Exame físico: "Bom estado geral" (B.E.G. ativo e reativo), hipocorado, acianótico, anictérico, afebril, eupneico e sem sinais de desconforto respiratório com $\mathrm{Sat}_{2}=98 \%$. Percussão com som claro pulmonar. Ausculta sibilante em bases pulmonares e roncos esparsos, em hemitórax esquerdo (HTE). Exame espirométrico com normalidade dos parâmetros, com parcial variação de volume após o uso de broncodilatador. A tomografia computadorizada de alta resolução (TCAR) de tórax apresentou áreas de consolidação no espaço aéreo, bilaterais e assimétricas com predominância nas regiões peribronquicas, algumas assumindo aspecto de nódulos pulmonares circundadas por vidro fosco. Posteriormente, no diagnostico diferencial, a tomografia computadorizada por emissão de
\end{abstract}


pósitrons (PET-CT) revelou um processo inflamatório em fase resolutiva, descartando outras doenças, por exemplo, o adenocarcinoma pulmonar. Optou-se pela prescrição de Prednisona e o acompanhamento do quadro com Radiografias de tórax que, após 4 meses, mostrava padrões dentro da normalidade. Atualmente o paciente se encontra bem e com remissão total dos sintomas e sob terapêutica contínua.

Palavras-chave: Bronquiolite obliterante; Adenocarcinoma; Diagnóstico diferencial; Opacidades isoladas; BOPO.

\begin{abstract}
Bronchiolitis obliterans organizing pneumonia (BOOP) is an entity with suggestive clinical and imaging diagnosis associated with evidence of connective tissue on the lumen of the distal air spaces at the lungs. Here we address a case of BOOP and highlight the importance of differential diagnosis. Male patient, 76 years old, ex-smoker (interrupted the habit about 30 years ago), developed complaints of copious and non-productive cough, thermometer fever of $38{ }^{\circ} \mathrm{C}$ and weight loss without relief from the use of fenoterol + ipatropium bromide. There was no recent history of infection, use of systemic or inhaled drugs, or travel. He reported a relevant family history for cancer. Physical examination: patient conscious and comfortable, pale, acyanotic, anicteric, afebrile, eupneic and without signs of respiratory distress with Sat $\mathrm{O}_{2}=98 \%$. Percussion with clear pulmonary sound. Hissing auscultation in pulmonary bases and sparse snoring in the left hemithorax. Spirometric examination with normal parameters, with partial volume variation after the use of bronchodilator. The high-resolution computed tomography (HRCT) of the chest showed areas of consolidation in the alveolar air space, bilateral and asymmetric, with predominance in the peribronchial regions, some assuming the appearance of pulmonary nodules surrounded by ground glass. Later, in the differential diagnosis, positron emission tomography-computed tomography (PET-CT) revealed an inflammatory process in the resolving phase, ruling out other diseases, for example, pulmonary adenocarcinoma. We opted for the prescription of Prednisone and the follow-up of the condition with chest X-rays, which, after 4 months, showed patterns within the normal range. Currently, the patient is "well" and with total remission of symptoms and under continuous therapy.
\end{abstract}

Keywords: Bronchiolitis obliterans; Adenocarcinoma; Differential diagnosis; Isolated opacities; BOOP.

\begin{abstract}
Resumen
La bronquiolitis obliterante con neumonía organizada (BONO) es una entidad con diagnóstico clínico y de imagen sugerente asociado con evidencia de tejido conectivo en la luz de los espacios aéreos distales de los pulmones. Aquí abordamos un caso de BONO y destacamos la importancia del diagnóstico diferencial. Paciente de sexo masculino, 76 años, exfumador (descontinuó el hábito hace unos 30 años) que desarrolló quejas de tos copiosa e improductiva, fiebre termométrica de $38{ }^{\circ} \mathrm{C}$ y pérdida de peso sin alivio por el uso de fenoterol + bromuro de ipatropio. Paciente sin antecedentes recientes de infección, uso de fármacos sistémicos/inhalados o viajes. Historial familiar relevante informado de cáncer. Examen físico: "buen estado general" (B.E.G. activo y reactivo), pálido, acianótico, anictérico, afebril, eupneico y sin signos de dificultad respiratoria con $\mathrm{Sat} \mathrm{O}_{2}=98 \%$. Percusión con claro sonido pulmonar. Auscultación de sibilancias en bases pulmonares y ronquidos escasos en hemitórax izquierdo. Prueba de espirometría con parámetros normales, con variación parcial de volumen tras el uso de broncodilatadores. La tomografía computarizada de alta resolución (TCAR) de tórax mostró áreas de consolidación en el espacio aéreo, bilaterales y asimétricas, de predominio en las regiones peribronquiales, algunas de las cuales tomaron la apariencia de nódulos pulmonares rodeados de vidrio deslustrado. Posteriormente, en el diagnóstico diferencial, la tomografía computarizada por emisión de positrones (PET-CT) reveló un proceso inflamatorio en fase de resolución, descartando otras enfermedades, por ejemplo, adenocarcinoma pulmonar. Decidimos prescribir prednisona y hacer seguimiento con radiografías de tórax que, a los 4 meses, mostraron patrones dentro de la normalidad. Actualmente, el paciente se encuentra bien y con remisión completa de los síntomas y en terapia continua.
\end{abstract}

Palabras clave: Bronquiolitis obliterante; Adenocarcinoma; Diagnóstico diferencial; Opacidades aisladas; BONO.

\title{
1. Introdução
}

A bronquiolite obliterante com pneumonia em organização (BOPO) é uma doença com manifestações clínicas, radiológicas e prognóstico peculiares, mas que podem se assemelhar a outras alterações patológicas intersticiais pulmonares (de Almeida et al., 2002; Addor et al., 2004; Cordier, 2006; Fonseca et al., 2007; Moraes et al., 2021). A BOPO é uma doença reportada mundialmente, com aproximadamente 1,6 casos para 100.000 habitantes e entre 6-7 casos para 100.000 internações, responsável por aproximadamente 20\%-30\% dos casos de doenças pulmonares infiltrantes (Cazzato et al., 2000; Alsaghir et al., 2007). A maioria dos pacientes afetados pela BOPO encontram-se na 
faixa etária dos idosos, entre 50-70 anos, embora também existam relatos de ocorrência na infância, não havendo predominância por sexo (de Almeida et al., 2002; Addor et al., 2004). Quanto a etiologia, a literatura aborda o desenvolvimento primário de BOPO ou como uma forma secundária a outras patologias e condições clínicas, devido a diferentes causas, incluindo: BOPO pós-infecção ou pós-transplante de órgãos; condições reumatológicas; exposição ambiental/ocupacional (na maioria dos casos, não relacionada ao tabagismo) ou uso crônico de medicamentosa/drogas de abuso e; em pacientes imunocomprometidos, neste caso, podendo a BOPO ocorrer por infecções oportunistas, como exemplo, por Aspergillus fumigatus, ou em pacientes sob radioterapia (Jhayya et al., 2000; de Almeida et al., 2002; Addor et al., 2004; Oymak et al., 2005; Cornelissen et al., 2007).

Primeiramente descrita por Epler et al. (1985), a BOPO apresenta na patogenia e prognóstico aspectos que destacam a importância de um diagnóstico diferencial, por exemplo, diferenciando-a de uma forma idiopática anteriormente descrita, nomeada pneumonia criptogênica em organização (PCO) (Davison et al., 1983; Akira et al., 1998; Lazoir et al., 2000; de Almeida et al., 2002; Addor et al., 2004; Cordier, 2006). Além disso, outras patologias e alterações do pulmão podem cursar com um quadro clínico/por imagem e perfil histopatológico semelhante, incluindo: neoplasia pulmonar, sarcoidose, doenças granulomatosas e tuberculose, outras doenças infecciosas, incluindo a causada pelo novo coronavírus (COVID-19, do inglês Coronavirus Disease-2019), vasculites, pneumonite de hipersensibilidade, pneumonia obstrutiva, pneumonia eosinofílica crônica, malignidades hematológicas, além de outras alterações pulmonares por aspiração, uso de drogas, toxinas sistêmicas ou inaladas (Addor et al., 2004; Tse et al., 2004; Oymak et al., 2005; Fonseca et al., 2007; Kory \& Kanne, 2020). A BOPO revela a presença de tecido conjuntivo no interior dos espaços aéreos distais, principalmente nos ductos alveolares e alvéolos e, com considerável frequência, acometem os bronquíolos, funcionalmente limitando sobremaneira o fluxo aéreo e levando a variados graus de disfunção respiratória (de Almeida et al., 2002; Addor et al., 2004; Oymak et al., 2005).

Neste relato de caso abordamos o diagnóstico de uma BOPO em um paciente idoso de risco, destacando a importância de um diagnóstico diferencial na prática médica, a fim de elencarmos as principais patologias as quais se deve dar importância para o quadro, e visando um diagnóstico concreto da doença, em tempo hábil para um prognostico o mais favorável possível.

\section{Relato do Caso}

Paciente JDP, sexo masculino, 76 anos, ex-tabagista (50 maços/ano), tendo interrompido o hábito há cerca de 30 anos, apresentou-se em 2018 a uma unidade ambulatorial de um hospital no sul de Minas Gerais, Brasil, com queixas de tosse copiosa e não produtiva há 3 meses sem alívio ao uso de fenoterol + brometo de ipatrópio, emagrecimento de aproximadamente $2 \mathrm{Kg}$ nesse período e febre termometrada de $38^{\circ} \mathrm{C}$. Paciente sem histórico recente de infecção, uso de drogas sistêmicas ou inaladas, ou viagem. Negava história de asma e sinusopatia. Refere história familiar positiva para diversas neoplasias (irmão e pai: câncer de cólon; 3 irmãs: câncer de mama) e história patológica pregressa de infarto agudo do miocárdio (IAM) há 30 anos.

Ao exame físico apresentava "bom estado geral" (B.E.G.), hipocorado, acianótico, anictérico, afebril, eupneico e sem sinais de desconforto respiratório, $\mathrm{Sat}_{2}=98 \%$. À percussão, som claro pulmonar. À ausculta sibilante em bases pulmonares e roncos esparsos, em hemitórax esquerdo (HTE).

Devido ao quadro, foi prescrito Ceftriaxona IM $1 \mathrm{~g}$ por 7 dias, ANORO® (umeclidinium e vilanterol). Além disso foi solicitado exames laboratoriais, espirometria e tomografia computadorizada de alta resolução (TCAR) de 
tórax. O resultado da espirometria encontrava-se dentro dos parâmetros de normalidade (Tabela 1) com variação parcial de volume em resposta ao broncodilatador (BD), em uso contínuo de $\mathrm{ANORO}^{\circledR}$.

Tabela 1 - Exame espirometrico mostrando normalidade nos parâmetros estudados e variação parcial de volume após uso de broncodilatador.

\begin{tabular}{|c|c|c|c|c|c|c|c|c|c|c|}
\hline \multicolumn{4}{|c|}{ F zE data da Curva 04/07/2018 } & \multicolumn{7}{|c|}{ POS broncodilatador com Salbutamol - 14:24:11 } \\
\hline Parametres & BTPS & Prev & PRE & Kerey & PQS & Sprev & \%Des & PRE\#1 & PRE 2 & PRERS \\
\hline \multicolumn{11}{|c|}{ Capacidade Vital Forçada } \\
\hline \multicolumn{11}{|c|}{ Meithores valores de todas as curves } \\
\hline FVC & L & 2.71 & 3,91 & 144 & 4.20 & 155 & 7 & 3,91 & 3,85 & \\
\hline FEV1 & L. & 2,11 & 3,36 & 160 & 3.37 & 160 & 0 & 3,36 & 3,22 & \\
\hline FEV1IFVC & $\%$ & 79.7 & 85,8 & 108 & 80,2 & 101 & -7 & 85,9 & 83,6 & \\
\hline PEF & L/s & 6.67 & 6,75 & 101 & 8,06 & 91 & -10 & 6,75 & 596 & \\
\hline \multicolumn{11}{|c|}{ Valores da melhor curva } \\
\hline FEF2575 & L/s & 2,76 & 3,65 & 169 & 3,47 & 761 & .5 & 3,66 & 3,318 & \\
\hline FEF25 & $\mathrm{U} / \mathrm{s}$ & 6,07 & 5,58 & 92 & 5,48 & 90 & -2 & 5,58 & 5,08 & \\
\hline FEF 50 & $\mathrm{L} / \mathrm{s}$ & 2,83 & 4,11 & 145 & 3.92 & 139 & -6 & 4,11 & 3.83 & \\
\hline FEF75 & Us: & 0.82 & 1,83 & 223 & 1.54 & 187 & -16 & 1,83 & 1,60 & \\
\hline Five & L & 2,71 & & & 0,00 & 0 & & & & \\
\hline Fiv2 & $L$ & 2.11 & & & 0.00 & 0 & & & & \\
\hline SVI/FIVC & $\%$ & 78,7 & 0,0 & o. & 0,0 & 0 & & 0,0 & 0,0 & \\
\hline E.A & Anos & 76 & 76 & & 76 & 100 & 0 & 76 & 76 & \\
\hline
\end{tabular}

Fonte: Autores.

TCAR de Tórax: atelectasia quase que completa do lobo inferior do pulmão esquerdo, associado à presença de focos consolidativos com broncogramas aéreos de permeio, circundados por opacidades em vidro fosco (Figura 1), com redução abrupta do calibre da porção proximal do brônquio para este lobo. Pequeno derrame pleural bilateral, associado a pequenas estrias atectásicas no parênquima pulmonar adjacente. Estrias fibroelásticas em lobo inferior do pulmão direito, leve ectasia do tronco da artéria pulmonar, podendo estar relacionado à hipertensão pulmonar. 
Figura 1 - Tomografia computadorizada de alta resolução (TCAR) de tórax mostrando áreas de consolidação alveolar, algumas assumindo aspecto de nódulos pulmonares, circundados por áreas com aparência de vidro fosco.

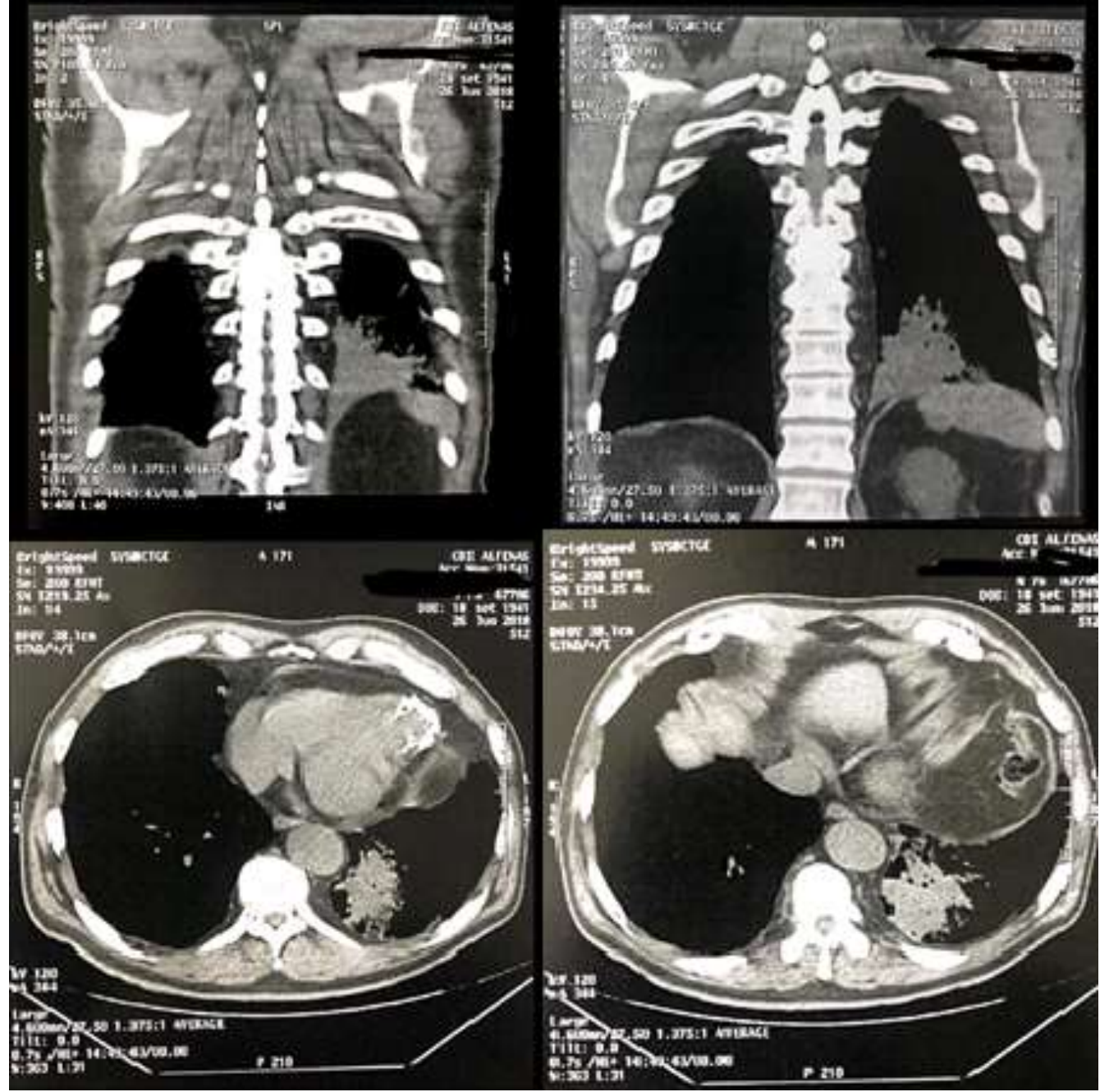

Fonte: Autores.

Com os achados da TCAR de Tórax, somado à clínica de consumpção e tosse persistente com discreto fator de melhora ao uso de broncodilatadores (umeclidinium e vilanterol - ANORO $^{\circledR}$ ), especulou-se, a princípio, o diagnóstico de um possível adenocarcinoma pulmonar e decidiu-se por solicitar a tomografia computadorizada por emissão de pósitrons (PET-CT) para estadiamento do mesmo. No entanto, na PET-CT, sob a fusão de imagens observou-se uma hipercaptação do radiofármaco (F18) em pequena consolidação parenquimatosa com componente atelectásico no lobo inferior do pulmão esquerdo ( $\mathrm{SUV}=4,3$ ). A interpretação foi de uma pequena consolidação parenquimatosa hipermetabólica no lobo inferior do pulmão esquerdo, devendo corresponder à processo inflamatório/infeccioso em fase resolutiva (Figura 2). Em comparação com o exame tomográfico houve significativa redução nas dimensões da opacidade descrita.

Com a conclusão do diagnóstico de um quadro inflamatório infeccioso, decidiu-se pela prescrição de Prednisona $10 \mathrm{mg}$ por 4 meses e o acompanhamento do quadro com Radiografias de tórax. No final de 4 meses, a não ser para os achados de doença pulmonar obstrutiva crônica (DPOC), a radiografia mostrava padrões dentro da normalidade. Atualmente o paciente se encontra bem e com remissão total dos sintomas e terapêutica contínua com Reovar Ellipta $^{\circledR}$ (Fuorato de flucasona e vilanterol), Montelucaste e Polaramine ${ }^{\circledR}$ (Dexclorfeniramina). 
Figura 2 - Tomografia Computadorizada por emissão de pósitrons (PET-CT), mostrando processo inflamatório/infeccioso em fase resolutiva.

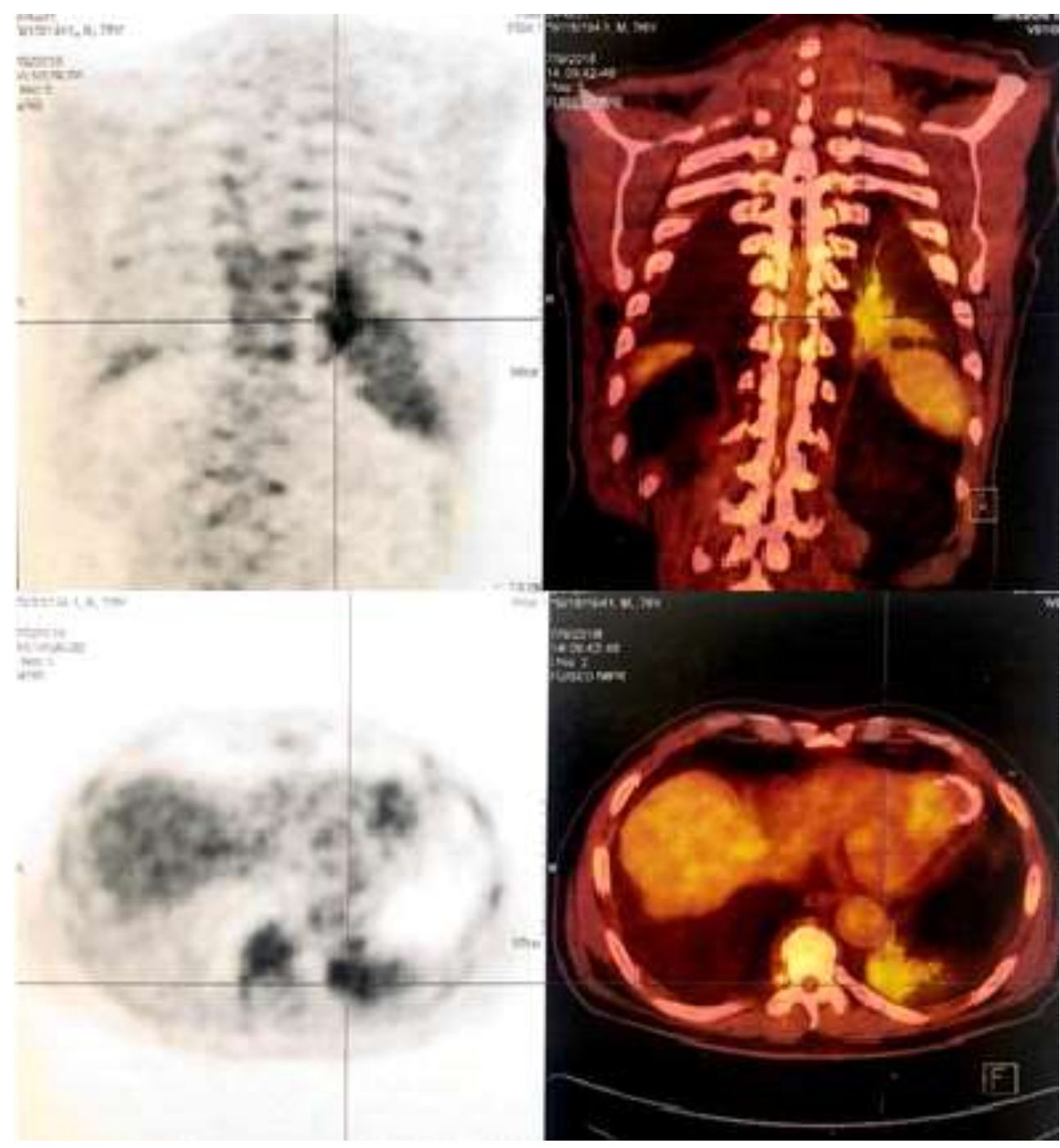

Fonte: Autores.

\section{Discussão}

A BOPO é, a princípio, uma patologia benigna, ocorrendo normalmente em formas subaguda ou crônica, contudo, existem condições em que esta doença se apresenta na forma mais aguda, podendo tornar-se potencialmente letal, sendo uma apresentação mais rara (de Almeida et al., 2002). Mesmo nas formas benignas, deve ser instituído o tratamento o mais rápido para uma maior eficácia clínica. Para tanto, devido a uma variada gama de diagnósticos diferenciais, é importante direcionar os exames complementares de acordo com as hipóteses, visando um diagnóstico preciso o mais rápido possível (Kitaichi, 1992; Akira et al., 1998; Addor et al., 2004; Muller et al., 2004; Drakopanagiotakis et al., 2008). A abordagem do clínico para tal, bem como as características clinicas em indivíduos com BOPO se mostram com um padrão clínico semelhante, por exemplo, ao da pneumonia aguda infecciosa ou subaguda infecciosa clássica (Akira et al., 1998), com sintomas comumente clássicos: tosse seca, febre, dispnéia, podendo também ocorrer sintomas constitucionais, como perda de peso, mialgia e prostração (de Almeida et al., 2002); sendo estes, os sintomas típicos observados no paciente do presente relato, durante admissão. As crepitações 
inspiratórias aparecem em grande parte dos casos, sendo um achado comum no exame físico (Akira et al., 1998; Muller et al., 2004; Drakopanagiotakis et al., 2008). Neste contexto, o direcionamento do diagnóstico deve ser imediato, pois apesar da BOPO apresentar geralmente um bom prognóstico, pode haver uma variante aguda, com sério risco de vida pela síndrome associada (Muller et al., 2004).

Quanto ao paciente do presente relato, as evidências no exame físico se mostraram distintas e ele apresentava-se em B.E.G., hipocorado, acianótico, anictérico, afebril, eupneico e sem sinais de desconforto respiratório. Devido a este quadro, naquele momento foi prescrito Ceftriaxona IM 1g por 7 dias, ANORO $^{\circledR}$ (umeclidinium e vilanterol) e solicitado exames laboratoriais, espirometria e TCAR de tórax.

Marcadamente, achados da BOPO em radiografias de tórax e na TCAR revelam uma consolidação multifocal, e radiografias em série mostram uma natureza migratória desta, com possíveis infiltrados também relacionados ao quadro (Kitaichi, 1992; Akira et al., 1998; Muller et al., 2004; Addor et al., 2004). No presente relato, a TCAR mostrou anormalidades semelhantes à radiografia de tórax. Essas anormalidades são inespecíficas na maioria dos casos, assim sendo, outras doenças que apresentam anormalidades semelhantes a BOPO devem ser consideradas quanto ao diagnóstico diferencial (Addor et al., 2004). Alguns pacientes com BOPO podem apresentar em radiografias, opacidades reticulares subpleurais periféricas, mas este achado pode estar presente, também, em outras alterações patológicas, tais como a doença intersticial e a fibrose (Akira et al., 1998; Santos et al., 2003). A presença de um nódulo ou massa pulmonar solitária que pode simular carcinoma, pode também estar presente na BOPO, além de outras patologias, levando a biópsia ou ressecção (de Almeida et al., 2002; Akira et al., 1998).

Destacando a importância do diagnóstico diferencial, a BOPO apresenta considerável similaridade com diversas doenças e condições pulmonares, como neoplasia pulmonar, sarcoidose, doenças granulomatosas e tuberculose, vasculites, outras doenças infecciosas (incluindo COVID-19), pneumonite de hipersensibilidade, pneumonia obstrutiva, pneumonia eosinofílica crônica, malignidades hematológicas, além de alterações pulmonares por aspiração, uso de drogas, toxinas sistêmicas ou inaladas (de Almeida et al., 2002; Santos et al., 2003; Addor et al., 2004; Tse et al., 2004; Kory \& Kanne, 2020). O caso aqui descrito revela a exclusão de um possível diagnóstico diferencial de adenocarcinoma pulmonar, através de um exame não invasivo e de alta acurácia para confirmação do quadro (PET-CT) (Fonti et al., 2019). O exame de escolha para a confirmação diagnóstica, normalmente, seria o estudo histopatológico da lesão, através da broncoscopia com biópsia, no entanto, devido às comorbidades do paciente [cardiopatia, idade avançada, e paciente sob terapia com anticoagulação contínua (Rivaroxabana)], optou-se pela PET-CT. Através deste diagnóstico por imagem, com SUV $=4,3$, descartou-se neoplasia, indicando na verdade tratar-se de um quadro inflamatório e infeccioso em fase resolutiva.

O tratamento da BOPO depende da causa e, geralmente, a corticoterapia é aconselhada. Nos casos subagudos ou crônicos, inicia-se geralmente prednisona na dose de $1 \mathrm{mg} / \mathrm{kg} / \mathrm{dia}$ por um a três meses; sendo a dose diminuída lentamente durante quatro a seis meses se o paciente está estável ou com melhora, reintroduzindo a terapia agressivamente ao sinal de recorrência. Quanto aos casos agudos, normalmente inicia-se metilprednisolona em altas doses $(0,5 \mathrm{a} 1 \mathrm{~g} / \mathrm{dia})$ por três a cinco dias, seguida de seis a 12 meses de corticoide oral. No paciente descrito no presente relato, decidiu-se pela prescrição de Prednisona $10 \mathrm{mg}$ por 4 meses e o acompanhamento do quadro com Radiografias de tórax. No final de 4 meses, a não ser achados de DPOC, a radiografia mostrava padrões dentro da normalidade. A BOPO é caracterizada pela rápida resolução do foco sob uso de corticosteroides, contudo, recidivas podem ocorrer por intercorrências ou não adesão ao tratamento (Addor et al., 2004). Nos casos de deterioração clínica sob tratamento com corticoide deve-se iniciar um agente citotóxico, geralmente, ciclofosfamida. Cerca de dois terços dos pacientes com BOPO apresentam resolução completa do quadro clínico com o tratamento, um desfecho aqui observado. A base 
histopatológica de lesões nos pulmões em condições inflamatórias semelhantes também são orientadoras para a instituição da corticoterapia em doenças semelhantes (Moraes et al., 2021); incluindo nas doenças causadas pelos

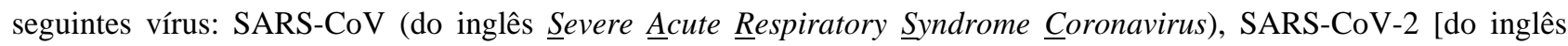
Severe Ácute Respiratory Syndrome Coronavirus-2) (doença conhecida como COVID-19) e MERS-CoV (do inglês

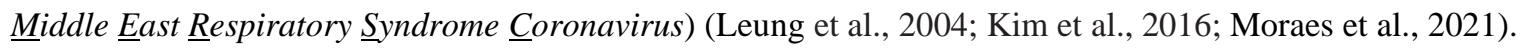

\section{Considerações Finais}

O presente relato evidencia a importância do diagnóstico diferencial na investigação da BOPO. O diagnóstico de outras doenças, por exemplo, o adenocarcinoma, foi aqui descartado através dos exames solicitados e clínica do paciente. Com os achados da TCAR de Tórax e, posteriormente na PET-CT (teoricamente para estadiamento de um possível adenocarcinoma), foi interpretada uma consolidação de um processo inflamatório onde foi descartada a hipótese de adenocarcinoma e constatou-se um processo inflamatório em fase resolutiva de uma BOPO. Após diagnostico diferencial e estabelecimento da BOPO, optou-se pela prescrição de Prednisona e o acompanhamento do quadro com Radiografias de tórax sendo que, após 4 meses, a radiografia mostrava padrões dentro da normalidade e atualmente o paciente se encontra bem e com remissão total dos sintomas e sob terapêutica contínua.

Como perspectivas, destaca-se o presente relato de caso apresentando um diagnóstico diferencial para a BOPO, utilizando as ferramentas clínicas, laboratoriais e de diagnóstico por imagem. Novos estudos e relatos de caso referentes a patogenia da BOPO, bem como referentes as estratégias diagnósticas, devem ser explorados, visando um melhor conhecimento acerca das diferenças entre as doenças pulmonares intersticiais, atualmente muito comum devido a pandemia de COVID-19, que em última análise, tais estudos podem lançar luz sobre a condução de melhores prognósticos bem como a obtenção de novas estratégias terapêuticas.

\section{Agradecimentos}

Gostaríamos de agradecer ao paciente e familiares, bem como a todos que contribuíram com o presente relato.

\section{Aspectos Éticos}

Os envolvidos diretos, paciente e familiares, foram esclarecidos sobre os propósitos do relato, oportunidade na qual foi assinado o TCLE. Este relato foi aprovado pelo comitê local de ética em pesquisa envolvendo humanos da UNIFENAS (parecer \#4.927.785).

\section{Referências}

Addor, G., Monteiro, A., Nigri, D.H, et al. (2004). Bronquiolite Obliterante com Pneumonia em Organização. J Bras Pneumol, $30(2), 154-157$.

Akira, M., Yamamoto, S., Sakatani, M. (1998). Bronchiolitis obliterans organizing pneumonia manifesting as multiple large nodules or masses. AJR, $170,291-295$.

Alsaghir, A. H., Al-Mobeireek, A. F., Al-Jahdali, H., et al. (2007). Bronchiolitis obliterans organizing pneumonia: experience at three hospitals in Riyadh. Ann Saudi Med, 27(1), 32-5.

Cazzato, S., Zompatori, M., Baruzzi, G., et al. (2000). Bronchiolitis obliterans-organizing pneumonia: an Italian experience. Respir Med, 94(7), 7028 .

Cordier, J-F. (2006). Cryptogenic organising pneumonia. Eur Respir J, 28, 422-446.

Cornelissen, R., Senan, S., Antonisse, I. E., et al. (2007). Bronchiolitis obliterans organizing pneumonia (BOOP) after thoracic radiotherapy for breast carcinoma. Radiation Oncology, 2, 2. 
Research, Society and Development, v. 10, n. 12, e120101220133, 2021

(CC BY 4.0) | ISSN 2525-3409 | DOI: http://dx.doi.org/10.33448/rsd-v10i12.20133

Davison, A. G., Heard, B. E., McAllister, W. A. C., et al. (1983). Cryptogenic organizing pneumonitis. Q J Med, 207, 382-394.

de Almeida, P., Guimarães, M. J. B., Costa, M. G., et al. (2002). Bronquiolite obliterante na forma nodular. J Pneumol, 28(6), 335-8.

Drakopanagiotakis, F., Polychronopoulos, L., Judson, M. A. (2008). Organizing pneumonia. The American Journal of the Medical Sciences, 355(1), 34-39.

Epler, G.R., Colby, T.V., McLoud, T.C., et al. (1985). Bronchiolitis obliterans organizing pneumonia. N Engl J Med, 312, 152-158.

Fonseca, L., Meruje, M., Barata, F., et al. (2007). Bronchiolitis obliterans organizing pneumonia - Experience of pulmonology Ward. Revista Portuguesa de Pneumologia, 13(3), 301-318.

Fonti, R., Conson, M., Del Vecchio, S. (2019). PET/CT in radiation oncology. Semin Oncol, 46(3), 202-209.

Jhayya, T. S., Perez, D. B., Llarges, C. M., et al. (2000). Bronquiolite obliterante com pneumonia em organização e aspergiloma em paciente com linfoma-leucemia de células T. J Pneumol, 26(1), 52-54.

Kitaichi, M. (1992). Differential diagnosis of bronchiolitis obliterans organizing pneumonia. Chest, 102(1), 44S-49S.

Kim, I., Lee, J. E., Kim, K-H., et al. (2016) Successful treatment of suspected organizing pneumonia in a patient with Middle East respiratory syndrome coronavirus infection: a case report. J Thorac Dis, 8(10), E1190-E1194.

Kory, P., Kanne, J. P. (2020). SARS-CoV-2 organising pneumonia: 'Has there been a widespread failure to identify and treat this prevalent condition in COVID-19?'. BMJ Open Resp Res, 7, e000724.

Lazoir, R., Vandevenne, A., Pelletier, A., et al. (2000). Cryptogenic organizing pneumonia: characteristics of relapses in a series of 48 patients. Am J Crit Care Med, 162, 571-577.

Leung, C. W., Chiu, W. K. (2004). Clinical picture, diagnosis, treatment and outcome of severe acute respiratory syndrome (SARS) in children. Paediatric Respiratory Reviews, 5, 275-288.

Moraes, H. R. S., Barboza, M. H. D., Tucci, J. A., Neto, J. B., Carvalho, L. F., Cerdeira, C. D., Souza, M. M. M. H. (2021). Bronquiolite obliterante em paciente pediátrico associada com exposição à cocaína e outras drogas de abuso durante o período gestacional. Research, Society and Development, 10(9), e35210918021.

Muller, N., Fraser, R., Lee, K., et al. (2004). Doenças do Pulmão: Correlação Radiológica e Patológica. Ed. Revinter, $1^{\text {a }}$ edição.

Oymak, F., Demirbaş, S. H., Mavili, M. E., et al. (2005). Bronchiolitis obliterans organizing pneumonia: clinical and roentgenological features in 26 cases. Respiration, 72(3), 254-62.

Santos, M. L. O., Marchiori, E., Vianna, A. D., et al. (2003). Opacidades em vidro fosco nas doenças pulmonares difusas: correlação da tomografia computadorizada de alta resolução com a anatomopatologia. Radiol Bras, 36, 329-338.

Tse, G.M-K., To, K-F., Chan, P.K-S., et al. (2004). Pulmonary pathological features in coronavirus associated severe acute respiratory syndrome (SARS). J Clin Pathol, 57, 260-265. 